\title{
LA ATRIBUCIÓN DE LA FILIACIÓN DE LOS HIJOS A LA ESPOSA O COMPAÑERA DE LA MADRE EN LAS PAREJAS DE LESBIANAS Un estudio de la jurisprudencia reciente
}

\author{
Francisco Javier Jiménez Muñoz \\ Profesor Titular Acreditado \\ Departamento de Derecho Civil \\ Universidad Nacional de Educación a Distancia (UNED)
}

\begin{abstract}
Resumen
En el presente artículo analizaremos la evolución producida en los últimos tiempos, especialmente en el ámbito jurisprudencial, en el tratamiento de la posibilidad de atribución, en parejas matrimoniales o de hecho de lesbianas, de la filiación de los hijos de una mujer a su esposa o compañera sentimental, ya sea por medio del recurso al expediente de la adopción o, últimamente, de la filiación por naturaleza. En este ámbito varias resoluciones judiciales han sido especialmente destacadas y en cuyo análisis nos centraremos: respecto de la vía de la adopción, el Auto del Juzgado de Primera Instancia $n^{\circ} 3$ de Pamplona de 22 de enero de 2004, junto a las SSTC 198/2012, de 6 de noviembre, y 93/2013, de 23 de abril; y la STS 740/2013, de 5 de diciembre, respecto de la atribución de la filiación matrimonial por naturaleza y la posibilidad del recurso a las acciones de filiación. No obstante, aún quedan ámbitos en que la equiparación entre parejas hetero y homosexuales no es total.
\end{abstract}

Palabras clave: parejas de lesbianas, matrimonio, parejas de hecho, filiación, adopción.

\begin{abstract}
In this article we will discuss the developments in recent times, especially in the field of jurisprudence, to treat the possibility of attribution, in lesbian married or de facto couples, of the parentage on a woman's children to her wife or de facto spouse, either through the use of adoption or, lately, of parentage by nature. In this area several judgments have been especially prominent and whose analysis we will focus: about the path of adoption, the Order of the First Instance Court No. 3 of Pamplona, January 22, 2004, together with the Constitutional Court Judgments 198/2012, November 6, and 93/2013, April 23, and the High Court Judgment 740/2013, December 5, regarding the attribution of matrimonial parentage by nature and the possibility of recourse to the parentage actions. However, there are still areas where the equation between hetero- and homosexual couples is not total.
\end{abstract}

Keywords: lesbian couples, marriage, de facto couples, parentage, adoption. 


\section{INTRODUCCIÓN}

En este artículo pretendemos poner de manifiesto los mecanismos que se han seguido sucesivamente por la jurisprudencia de los últimos años en relación con las parejas (matrimoniales o no) de lesbianas para la atribución de la filiación sobre los hijos a la esposa o compañera sentimental de la madre. Aquí hemos de tener presente que empleamos el término madre en sentido amplio, abarcando tanto a quien lo es biológicamente (por medios naturales o por recurso a la aplicación de las técnicas de reproducción asistida) como consecuencia de un proceso de adopción.

De ahí la necesidad, para no extender excesivamente el título, de tener que emplear una fórmula sintética que precisa ahora de explicación. Estudiaremos así la situación que en la contemplación de la jurisprudencia más reciente tiene la relación filiativa del hijo de una madre biológica o adoptiva que está unida a otra mujer, por vínculos matrimoniales o como pareja de hecho, con dicha esposa o compañera de su madre, relación a la que se puede llegar como filiación por naturaleza (en el sentido entendido que veremos en su momento) o adoptiva. Podemos de este modo distinguir una filiación por naturaleza y adoptiva, y entre mujeres casadas o en unión de hecho, dando así lugar a cuatro posibles situaciones, agrupables fundamentalmente en torno a si la naturaleza de la filiación será adoptiva o por naturaleza.

Iniciaremos, pues, esta visión de la evolución de la jurisprudencia por el tratamiento dado a la adopción por la pareja de hecho de la madre.

\section{LA ADOPCIÓN POR LA PAREJA MATRIMONIAL O DE HECHO DE LA MADRE}

\subsection{La adopción en parejas de hecho de lesbianas. El Auto del Juzgado de Primera Instancia $n^{\circ} 3$ de Pamplona de 22 de enero de 2004 y las leyes autonómicas de parejas de hecho}

En este punto, debemos empezar por el Auto del Juzgado de Primera Instancia $n^{\circ} 3$ (Familia) de Pamplona de 22 de enero de 2004, que si bien ciertamente no crea jurisprudencia sí ha sido una de las resoluciones judiciales más polémicas de los últimos años, al tratarse de la primera resolución judicial en nuestro país que otorga la patria potestad compartida a dos madres, la biológica y la adoptiva. En ella, se determina la atribución de la filiación sobre dos gemelas a la compañera sentimental de la madre biológica de las niñas, sobre la base de la adopción.

En este caso, ambas mujeres llevaban formando una unión de hecho desde hace más de siete años, si bien convivían desde 1999. En 2000 se propusieron como objetivo común la maternidad, y acudieron a tal efecto a un Centro de Planificación Familiar, buscando acceder a dicha maternidad por medio de técnicas de reproducción asistida, 
en cuya gestión resultaron favorables los informes de valoración psicológica tanto individuales como de pareja. Intentadas por una de las mujeres las técnicas de inseminación artificial y fecundación in vitro, sin embargo, no terminaron en gestación, por lo que fue posteriormente su compañera la que con éxito se sometió a las mencionadas técnicas que dieron lugar al nacimiento de las gemelas. La primera instó la adopción de las niñas, concediéndose por el citado Auto en aplicación de la Ley Foral $6 / 2000$ de 3 de julio, para la igualdad jurídica de las parejas estables y sobre la base del interés superior de las menores.

El Auto entiende que, dado que el artículo 8 de la referida Ley Foral establece que "los miembros de la pareja estable podrán adoptar de forma conjunta con iguales derechos y deberes que las parejas unidas por matrimonio", ha de concluirse la admisión de la adopción conjunta por quienes integren una pareja de hecho con independencia de su identidad sexual.

Ello podría interpretarse en el sentido de que, al momento en el que se dictó el Auto, se admitía la adopción conjunta, realizada simultáneamente por ambos miembros de la pareja, pero no la sucesiva, y por tanto se impediría que la compañera de la madre biológica de las menores pudiera adoptarlas, pues ha de tenerse en cuenta que en el Derecho común la Disposición Adicional Tercera de la Ley 21/1987, de 11 de noviembre ("las referencias de esta ley a la capacidad de los cónyuges para adoptar simultáneamente a un menor será también aplicables al hombre y a la mujer integrantes de una pareja unida de forma permanente por relación de efectividad análoga a la conyugal"), y los artículos $178.2 .1^{\circ}$ y $2^{\circ}$ del Código Civil permitían la adopción conjunta pero dejando cerrada la posibilidad de adopción sucesiva en parejas homosexuales, por el compañero del padre o por la compañera de la madre de los hijos de aquél o de ésta, con la consiguiente extinción de la filiación del otro padre o madre, en la medida en que tales preceptos sólo admiten al cónyuge del padre o madre (biológico o adoptivo) del adoptando, o al conviviente $\mathrm{u}$ otra persona de sexo diferente al progenitor cuando se hubiera determinado la filiación sólo con éste, y en ambos casos, sin extinguirse los vínculos con la familia anterior. De este modo, podría adoptar una persona sola, un matrimonio o una pareja de hecho heterosexual, pero no los miembros de una pareja homosexual (por ello, para Vallés, 2005, debería haberse denegado esta adopción, pues la extensión de la aplicación de los citados preceptos por las diversas decisiones judiciales lo ha sido a las uniones extramatrimoniales de carácter heterosexual; también Gavidia, 2001, consideraba que en estos casos se produciría la exclusión de la adopción conjunta por parejas integradas por personas del mismo sexo, y que esa exclusión no sería discriminatoria). Con la posterior reforma del artículo 178 por la Ley 13/2005, de 1 de julio, por la que se modifica el Código Civil en materia de derecho a contraer matrimonio, el requisito de que el adoptante sea de distinto sexo al del progenitor cuya filiación se haya determinado ha sido suprimido, y por tanto el obstáculo a la adopción de los hijos de un miembro de la pareja de hecho, homosexual o no, siempre que sólo aparezca determinada la filiación del otro miembro (lo que en el caso de las parejas homosexuales, en que es frecuente el recurso a las técnicas de reproducción asistida, será habitual).

Sin embargo, entiende el Auto que tampoco se produciría esa exclusión pues el impedimento legislativo (hoy ya inexistente en ese sentido, como hemos indicado) no 
tendría por objeto los supuestos en que la adopción por el compañero o compañera deviene de una previa situación de paternidad o maternidad individual del otro, y por tanto considera que si el legislador navarro está permitiendo la adopción conjunta por parejas homosexuales, difícilmente se podría encontrar sentido a una exclusión de la adopción por el compañero/a del padre o madre adoptivo, del hijo/a/s de éste, o por la compañera de la madre biológica, del hijo/a/s de ésta, en tales supuestos de maternidad o paternidad previa individual, cuando para acceder a ésta la propia legislación común lo permite con independencia de la identidad sexual o convivencia con pareja del mismo sexo. Asimismo, la jurisprudencia de las Audiencias ha realizado una interpretación favorable a la asimilación plena a estos efectos entre parejas matrimoniales y de hecho, sobre la base de ser ese el espíritu de la Ley (como la SAP Valladolid de 12 de junio de 2000) o el beneficio del adoptando (así, la SAP Barcelona de 30 de diciembre de 2002), si bien es cierto que aquí el Auto hace trampa con esas citas, pues las referidas sentencias se referían a parejas de hecho heterosexuales (Vallés, 2005).

En cualquier caso, una vez admitida la posibilidad de adopción sucesiva de los hijos menores por la mujer pareja de hecho de la madre, es claro que no será necesaria la propuesta de la entidad pública competente, conforme al artículo $176.2 .2^{\circ}$ del Código Civil, tanto por la citada equiparación a estos efectos entre parejas matrimoniales y de hecho como por la interpretación amplia del término consorte de dicho precepto que realizan las Audiencias (por los mismos motivos, ya que la extensión conceptual quedaría restringida a las parejas heterosexuales, Vallés, 2005, considera en cambio que sí debería haber intervenido la entidad pública correspondiente).

Finalmente, destaca el Auto que ha de tenerse presente en todo caso como primordial consideración el interés superior del niño, en especial en la adopción (artículos 3.1 y 21.1 de la Convención de las Naciones Unidas sobre los Derechos del Niño de 1989), y por tanto lo que debe ser objeto de análisis a la hora de decidir por el Juzgador acerca del establecimiento de una filiación adoptiva debe ser la idoneidad del adoptante en relación con el interés del adoptando y en su adecuación para procurarle los cuidados y educación necesarios para su desarrollo integral, conforme a los criterios establecidos por la Convención de Estrasburgo de 1967 sobre adopción de niños: la personalidad, la salud y situación económica del adoptante, su vida familiar y su aptitud para educar al adoptando; los motivos por los que el adoptante desea adoptar al niño, la relación entre adoptante y adoptando y la duración del período en el que el adoptando fue confiado al cuidado del que quiere adoptarlo, y la personalidad y salud del adoptando, sus orígenes y demás circunstancias culturales si procedieren, lo que en el caso enjuiciado se ha puesto de manifiesto favorablemente.

Si la Ley Foral navarra podía llevar a considerar interpretativamente admisible la adopción que estamos considerando entre las mujeres constituidas en pareja de hecho, como hace el Auto analizado, ello es aún más claro con la Ley vasca 2/2003, de 7 de mayo, que expresamente contempla tal posibilidad, tanto en relación con las adopciones conjuntas como sucesivas. Así, mientras que su artículo 8.1 establece que "Los miembros de parejas formadas por dos personas del mismo sexo podrán adoptar de 
forma conjunta, con iguales derechos y deberes que las parejas formadas por dos personas de distinto sexo y las parejas unidas por matrimonio", respecto de la adopción sucesiva el apartado 2 del mismo precepto establece que "La hija o hijo adoptivo o biológico de una de las partes de la pareja tendrá derecho a ser adoptado por la otra parte" (si bien esta disposición fue objeto de recurso de inconstitucionalidad por el Gobierno, el mismo fue objeto de desistimiento, acordándose su declaración por ATC de 14 de diciembre de 2004).

Las otras leyes autonómicas que regulan las parejas de hecho no contemplan la posibilidad de adoptar (a lo sumo, la posibilidad de recurrir al acogimiento en sus modalidades simple y permanente, pero no preadoptiva: así, las Leyes asturiana 4/2002, de 23 de mayo, de parejas estables; andaluza 5/2002, de 16 de diciembre, de parejas de hecho; y extremeña 5/2003, de 20 de marzo, de parejas de hecho de la Comunidad Autónoma de Extremadura). No obstante, tanto la interpretación extensiva de los términos cónyuge y consorte como la aplicación del actual artículo $178.2 .2^{\circ}$ del Código Civil, a que nos referimos con anterioridad, permiten entender que se abre con carácter general la posibilidad de la adopción por la compañera de la madre biológica o adoptiva que estamos considerando siempre que sólo se haya determinado la filiación.

Por su parte, los artículos 115.2 y 117.1.a) del Código de Familia de Cataluña, que contemplaban la posibilidad de adopción por más de una persona en el caso de cónyuges o parejas estables, desde su reforma por la Ley catalana 3/2005, de 8 de abril, no señalan ya si se trata de parejas homosexuales o heterosexuales (antes se hacía referencia respectivamente a la "pareja de hombre y mujer que convivan maritalmente con carácter estable" y a la "persona de sexo distinto con quien el adoptante convive maritalmente con carácter estable"), y por tanto claramente admiten la adopción, conjunta o sucesiva, tanto por parejas de lesbianas de naturaleza matrimonial como de hecho. Ello se mantiene con su respectiva integración, como artículos 235-30.2 y 235-32.1.a), en el Libro Segundo del Código Civil de Cataluña (Ley 25/2010, de 29 de julio).

\subsection{El Tribunal Constitucional}

También el Tribunal Constitucional se ha pronunciado sobre la posibilidad de que las parejas del mismo sexo adopten a menores.

En primer lugar, y en relación con los matrimonios de cónyuges del mismo sexo, podemos citar la STC 198/2012, de 6 de noviembre, que resuelve el recurso de inconstitucionalidad en relación con la Ley 13/2005, de 1 de julio, por la que modifica el Código civil en materia de derecho a contraer matrimonio, que venía a instaurar el matrimonio entre personas del mismo sexo. En concreto, se pronuncia sobre esta cuestión en su Fundamento Jurídico 12, en cuanto que la nueva redacción del apartado 4 del artículo 175 del Código Civil, aun sin aludir expresamente a la orientación sexual de los adoptantes, posibilita la adopción conjunta de menores por matrimonios entre personas del mismo sexo, lo que - se alega por los recurrentes - resultaría contrario al mandato de protección integral de los hijos (artículo 39.2 de la Constitución) al anteponer la legitimación de las relaciones homosexuales al interés del menor y a la idoneidad de los adoptantes. 


\section{LA ATRIBUCIÓN DE LA FILIACIÓN DE LOS HIJOS A LA ESPOSA O COMPAÑERA DE LA MADRE EN LAS PAREJAS DE LESBIANAS}

Al respecto, el Tribunal destaca el papel fundamental del interés del menor adoptado, que ha de ser preservado conforme al citado precepto constitucional tanto si es adoptado por un matrimonio entre personas del mismo sexo, como si lo es por un matrimonio entre personas de distinto sexo, pero ello ya se tutela en cada caso concreto en función del escrutinio al que se somete a los eventuales adoptantes con independencia de su orientación sexual, no quedando afectado el deber de protección integral de los hijos por el hecho de que se permita o se prohíba a las personas homosexuales adoptar, bien de forma individual, bien conjuntamente con su cónyuge. Así, el Tribunal Europeo de Derechos Humanos en su Sentencia de 26 de febrero de 2002, en el asunto Frette c. Francia, entiende que son las autoridades nacionales quienes, teniendo en cuenta los intereses de la sociedad y las sensibilidades y contextos locales, deberán apreciar con amplio margen la admisibilidad de la adopción por homosexuales, si bien teniendo presente que la adopción es dar una familia a un niño, y no un niño a una familia y el Estado debe asegurarse de que las personas elegidas como adoptantes sean las que puedan ofrecerle, desde todos los puntos de vista, las condiciones de acogida más favorables, y en este punto no existe certeza que permita afirmar actualmente que esas condiciones no puedan ser proporcionadas por una pareja homosexual.

Sólo si se demostrara que tener dos padres o dos madres es perjudicial para el menor, debería excluirse la adopción por parejas homosexuales, pues en tal caso efectivamente se estaría primando como consideración primordial no el interés superior del niño sino la equiparación de las parejas homosexuales a las otras (Gavidia, 2001). Salvo que se pruebe que ese perjuicio existe en todo caso, estas adopciones podrán ser admitidas y luego será en cada caso concreto el juez quien decida si las aprueba, en función de la idoneidad de los adoptantes (ibid.).

El mandato de protección a la familia en general (artículo 39.1 de la Constitución) y de los hijos en particular (artículo 39.2 de la Constitución) quedaría de este modo cubierto suficientemente con los mecanismos existentes para garantizar la preservación del interés superior del menor en el proceso de adopción y la idoneidad del adoptante o adoptantes para el ejercicio de la patria potestad, idoneidad que nada puede tener que ver con su orientación sexual, considerando además que el juez que conoce del proceso de adopción podrá denegarla cuando entienda que por cualquier motivo pueda ser contraria al interés del menor (STC 124/2002, de 20 de mayo). En todo caso, lo que en modo alguno resulta constitucionalmente admisible es presumir la existencia de un riesgo de alteración efectiva de la personalidad del menor por el mero hecho de la orientación sexual de uno u otro de sus progenitores (STC 176/2008, de 22 de diciembre, y STEDH de 30 de noviembre de 2010, asunto P.V. c. España) (Vallés, 2005, en cambio, considera que esta adopción puede producir graves consecuencias en la formación integral de las menores "al no distinguir la figura paterna y materna inherentes al ejercicio de la patria potestad, por cuanto en el entorno social de las adoptandas, éstas se sentirán diferentes en la configuración de su núcleo familiar al resto de las familias" y que el interés del menor "implica necesariamente reconocimiento social", 
argumento frente al que Durán, 2004, señala que " lo negativo, si lo hubiera, viene de fuera, de los prejuicios y de la falta de educación en la diversidad y en el respeto. Pero de las carencias de otros no se puede hacer responsables a estas familias").

Por otra parte, específicamente respecto de la legislación navarra, si bien la STC 93/2013, de 23 de abril, ha declarado inconstitucional y nulo parte del articulado de la Ley Foral 6/2000, los aspectos tenidos en cuenta en el Auto del Juzgado de Primera Instancia $n^{\circ} 3$ de Pamplona de 22 de enero de 2004, que hemos estudiado, no se han visto afectados, antes bien la resolución del Tribunal Constitucional se pronuncia expresamente (Fundamento Jurídico 12) reiterando los argumentos expuestos en la STC 198/2012, que acabamos de ver, en el sentido de rechazar que se anteponga al interés del menor el de las parejas homosexuales en adoptar, ni que el deber de protección integral de los hijos (artículo 39.2 de la Constitución) se vea afectado. Así, dichos argumentos, que la STC 198/2012 aplicó al matrimonio, ahora se generalizan al aplicarlo también a las uniones de hecho.

En cambio, en contra de la adopción por parejas homosexuales se ha pronunciado en sendos votos particulares el magistrado Juan José González Rivas, tanto en la STC 198/2012 como en la 93/2013, por entender que de los parámetros constitucionales expresamente prefijados en los artículos 32 y 39 de la Constitución se infiere la imposibilidad de desarrollar una relación de filiación adoptiva por dos adoptantes del mismo sexo, lo que sería concorde con la jurisprudencia del Tribunal Europeo de Derechos Humanos (así, cita las SSTEDH de 26 de febrero de 2002, asunto Fretté c. Francia; de 24 de junio de 2010, asunto Schalk y Kopf c. Austria; y de 30 de noviembre de 2010, asunto P.V. c. España).

\subsection{Conclusión: el actual reconocimiento de la posibilidad de adopción de los hijos por la mujer que sea la cónyuge o pareja de hecho de su madre biológica o adoptiva}

De este modo, inicialmente y hasta la Ley $13 / 2005$ podíamos considerar restringida la posibilidad de adopción en los términos que hemos visto, dada la exigencia de heterosexualidad de los artículos $178.2 .1^{\circ}$ y $2^{\circ}$ del Código Civil (al no existir matrimonio homosexual y requerirse la heterosexualidad para la adopción fuera el matrimonio), salvo en los casos de aplicación de las leyes vasca o navarra (o del Código de Familia catalán desde su reforma por la Ley 3/2005), si bien venía siendo cada vez más frecuente en las últimas resoluciones judiciales una interpretación extensiva de los conceptos de cónyuge y consorte a fin de integrar a las parejas de hecho y un ajuste interpretativo de ese requisito de heterosexualidad.

Sin embargo, actualmente y en especial desde la Ley $13 / 2005$ no existe ya obstáculo alguno a la adopción sucesiva por una mujer miembro de la pareja, matrimonial o de hecho, de los hijos de su cónyuge o compañera, madre biológica o adoptiva de los menores, lo mismo que no lo hay para una adopción conjunta o simultánea por ambas mujeres, pues estas adopciones deben considerarse equiparadas en todos los sentidos a las que puedan realizar las parejas heterosexuales. Por una parte, podemos encontrarnos ante un matrimonio, con lo que la adopción por una cónyuge de los hijos

Revista sobre la infancia y la adolescencia, 6, 51-63 - Marzo 2014

ISSN 2174- 7210 
de su esposa encontrará perfecta cabida en el artículo $178.21^{\circ}$ del Código; o por otra ante una pareja de hecho, supuesto que entraría en el artículo $178.22^{\circ}$ del mismo Código, que ya no requiere la diferencia de sexo entre el adoptante y el progenitor cuya filiación se mantiene.

Como mantiene el Tribunal Constitucional y parte de la doctrina, la orientación sexual del adoptante no es un dato relevante para la admisibilidad o no de una adopción concreta, sino que ésta ha de venir presidida en todo caso por el interés superior del menor y la idoneidad del adoptante o adoptantes para el ejercicio de la patria potestad, lo que el juez que conozca del proceso valorará en cada caso, denegando la adopción cuando por cualquier motivo la estime contraria al interés del menor.

\section{LA ATRIBUCIÓN DE UNA FILIACIÓN POR NATURALEZA A LA PAREJA MATRIMONIAL O DE HECHO DE LA MADRE. LA APLICACIÓN DE LAS TÉCNICAS DE REPRODUCCIÓN ASISTIDA}

\subsection{La atribución de la filiación por naturaleza en las parejas de lesbianas}

Hasta la reforma del concepto de matrimonio efectuada como consecuencia de la Ley $13 / 2005$, era clara la imposibilidad de la constitución de una relación filiativa por naturaleza para las parejas del mismo sexo, y específicamente de lesbianas, existiendo sólo la posibilidad de una adopción conjunta, o bien de una adopción sucesiva, por medio de la constitución inicial de la filiación de uno de los miembros de la pareja (por vía de adopción, concepción natural o recurso a las técnicas de reproducción asistida) y la posterior adopción del menor por la otra mujer miembro de la pareja. En todo caso, como vimos, esa admisibilidad no estaba exenta de problemas, quedando en gran medida limitada al ámbito de aplicación de las leyes de parejas de hecho vasca y navarra (ésta última por la vía de la interpretación) o bien restringida a los casos en que el juez realizaba un ajuste interpretativo de los requisitos legales para la adopción.

La reforma del Código Civil de 2005 hizo que el requisito de la heterosexualidad desapareciera de la institución matrimonial, abriéndose de este modo la puerta a la posibilidad de matrimonios entre personas del mismo sexo, y equiparándose consecuentemente la determinación de la filiación de las parejas homosexuales con la de las heterosexuales, pudiéndose así establecer una filiación por naturaleza en el caso de las parejas homosexuales (incluso no matrimoniales, dada la equiparación de las parejas de hecho a los matrimonios a estos efectos), en especial por cuanto el nuevo segundo párrafo del artículo 44 del Código establece que "el matrimonio tendrá los mismos requisitos y efectos cuando ambos contrayentes sean del mismo o de diferente sexo". Esa atribución de filiación es obviamente imposible en las parejas de varones, pero podría darse en el caso de parejas de mujeres, que podrían determinar una filiación matrimonial o no matrimonial respecto de un hijo que se hubiera concebido 
mediante el recurso a las técnicas de reproducción asistida.

La situación bajo la Ley 35/1988, de 22 de noviembre, sobre técnicas de reproducción asistida no permitía ese establecimiento de una filiación por naturaleza de la esposa o pareja femenina de la madre, dado que en su artículo 8 se hacía referencia expresa al marido y la mujer (salvo que interpretáramos extensivamente estos términos) y a efectos del juego de la presunción de paternidad (que por otra parte, sólo muy forzadamente se podía aplicar a la esposa de la madre) se consideraba como "escrito indubitado a los efectos previstos en el artículo 49 de la Ley del Registro Civil, el documento extendido ante el Centro o establecimiento autorizado, en el que se refleje el consentimiento a la fecundación con contribución del donante, prestado por varón no casado" (precisamente, en la tramitación parlamentaria de la Ley 13/2005 se excluyó la reforma de los artículos 48 y 49 de la Ley del Registro Civil en el sentido de admitir el reconocimiento como vía de determinación de la filiación en parejas del mismo sexo). Quedando así como única posibilidad, ante la falta de una clara presunción similar a la de paternidad del marido masculino, el recurso a la posterior adopción por la otra mujer del hijo concebido por su pareja o esposa por medio de las técnicas de reproducción asistida (Zurita, 2006; Hernández, 2007; Jiménez, 2008 y 2012).

Esta posición era mantenida también por la Dirección General de los Registros y del Notariado, que subrayaba el principio de la verdad biológica como fundamento de la filiación (como declaró el Tribunal Constitucional en sus Sentencias 273/2005, de 27 de octubre, y 52/2006, de 16 de febrero), y así en su Resolución de 5 de junio de 2006, sobre la pretensión de una pareja de hecho de lesbianas de inscribir a la compañera sentimental de la madre biológica que consintió su inseminación artificial, y en base a ese consentimiento, como madre de la hija nacida de la aplicación de las técnicas de reproducción asistida, se declara que la maternidad es única y viene determinada por el hecho del nacimiento, sin que pueda sobrevenir otro reconocimiento de maternidad por otra mujer, lo que sólo podría obtenerse a través de la adopción (abierta actualmente a las parejas del mismo sexo y que permite obtener la constitución de una relación jurídica de filiación de igual contenido, dado el principio de equiparación absoluta entre la filiación natural y la adoptiva), y sin que quepa la aplicación analógica de la antes citada presunción de que los hijos nacidos a consecuencia de la fecundación asistida de la madre se considerarán hijos del hombre que la ha consentido previamente en documento público.

Con la nueva Ley 14/2006, de 26 de mayo, sobre técnicas de reproducción humana asistida, el problema en gran medida se mantenía inicialmente, dado que buena parte de su contenido (y en concreto el de su artículo 7) era sustancialmente idéntico a la Ley de 1988, como destaca la Resolución de la Dirección General de los Registros antes citada. Ello hizo que por la Disposición Adicional primera de la Ley 3/2007, de 15 de marzo, reguladora de la rectificación registral de la mención relativa al sexo de las personas, hubiera de introducirse un nuevo apartado 3 , que viene a contemplar específicamente esta situación, al establecer que "cuando la mujer estuviere casada, y no separada legalmente o de hecho, con otra mujer, esta última podrá manifestar ante el Encargado del Registro Civil del domicilio conyugal, que consiente en que cuando nazca el hijo de su cónyuge, se determine a su favor la filiación respecto del nacido" (solución legislativa que critica Arechederra, 2006, puesto que al ser la reproducción 
esencialmente heterosexual, aquélla atentaría contra la dignidad del que va a nacer por cuanto le atribuiría una filiación imposible en términos biológicos que le discriminaría respecto a los demás nacidos, por lo que entiende que sería inconstitucional).

El problema se sigue planteando, pues, respecto de los casos en que ambas mujeres no están casadas sino que simplemente constituyen una pareja de hecho o en que la concepción se hayan seguido procedimientos distintos a las técnicas médicas de reproducción asistida (así, el recurso a un amigo varón para una concepción natural), en cuyo caso deberá seguir acudiéndose al procedimiento de adopción. Ello ha sido criticado por cuanto se estaría discriminando a las parejas de lesbianas (Benavente, 2011; Garaizabal et al., 2012): mientras que las parejas heterosexuales no tienen que estar casadas para poder reconocer a sus hijos y nadie les exige la existencia real de una relación biológica con los descendientes reconocidos por el varón, la determinación de la filiación por dos madres se subordina a que ambas estén casadas y hayan recurrido a las técnicas de reproducción asistida, que además en estos casos no entran en las prestaciones de la sanidad pública: por tanto, esta solución sólo serviría para las parejas que estén casadas y puedan asumir económicamente la aplicación de estas técnicas en la sanidad privada. Algunas de estas objeciones parecen haber quedado superadas interpretativamente con la Sentencia del Tribunal Supremo de 5 de diciembre de 2013, que veremos en el siguiente apartado.

En cambio, este problema no se da en Cataluña, donde el Libro Segundo del Código Civil de Cataluña contempla la posibilidad de la atribución de la filiación por naturaleza a las parejas de lesbianas de índole tanto matrimonial como no matrimonial. Así, el artículo 235-8, respecto de la filiación matrimonial, habla simplemente de cónyuge (que ha consentido a la fecundación asistida de su esposa), mientras que el 235-13.1, en relación con la filiación no matrimonial, señala que "los hijos nacidos de la fecundación asistida de la madre son hijos del hombre o de la mujer que la ha consentido" (estos artículos tienen su origen, respectivamente, en los artículos 92.1 y 97.1 del Código de Familia, en la redacción dada por la Ley 10/2008, de 10 de julio; antes se hablaba de marido y exclusivamente de hijos del hombre que la ha consentido).

\subsection{La Sentencia del Tribunal Supremo 740/2013, de 5 de diciembre}

Como vemos, la adquisición de la filiación por naturaleza cuando la madre y la otra mujer están casadas y se recurre a la aplicación de las técnicas de reproducción asistida es clara en la actualidad, tras la reforma del artículo 7 de la Ley 14/2006 por la Ley $3 / 2007$. Ello ha quedado reforzado con la reciente Sentencia de la Sala $1^{\text {a }}$ del Tribunal Supremo 740/2013, de 5 de diciembre, que, dando un paso más en este proceso evolutivo, declara la admisibilidad del ejercicio de acciones de filiación en el contexto de las parejas homosexuales, y destaca como elemento atributivo de la filiación el voluntario consentimiento para la técnica de reproducción asistida y la voluntad concorde de las partes de concebir un hijo, bastando con que ese consentimiento conste como consentimiento informado para la aplicación de las técnicas, más allá del 
requisito formal de que dicho consentimiento conste ante el encargado del Registro Civil, que queda así relativizado. Y es más, el requisito de la matrimonialidad en cierta medida se debilita al atenderse fundamentalmente a la existencia del consentimiento de ambas mujeres a la aplicación de las técnicas, bastando indirectamente con que la matrimonialidad concurra antes del nacimiento, y así en el caso que resuelve tal consentimiento se prestó antes del matrimonio, pero éste se celebró con anterioridad al nacimiento de las niñas (luego estaríamos en todo caso ante una filiación matrimonial).

En el caso enjuiciado en esta sentencia, una pareja de lesbianas tienen tres hijas en común, nacidas por aplicación de inseminación artificial, si bien el problema estudiado se centró en torno a las dos gemelas más pequeñas (la otra se inscribió como hija de su progenitora biológica, madre soltera, y fue adoptada por su compañera). Habiéndose iniciado el procedimiento de reproducción asistida con consentimiento informado firmado por las dos mujeres el 16 de marzo de 2007, ambas contrajeron matrimonio el siguiente 3 de agosto, produciéndose el nacimiento de las gemelas el 14 de diciembre, siendo inscritas en el Registro Civil únicamente como hijas de su progenitora biológica. Ésta inició expediente de rectificación de error de las inscripciones registrales, a efectos de que se rectifique el estado civil de la madre biológica que no es de soltera, sino casada, y para que se identifique a su cónyuge a los efectos de la patria potestad y designación de apellidos de las dos menores, expediente que acabó por auto que acordaba que debe rectificarse el estado civil de la madre y no accediendo al resto de las solicitudes interesadas. La progenitora interpuso contra dicho auto recurso que resultó resuelto por la DGRN en fecha 26 de noviembre de 2008 desestimándolo íntegramente. En junio de 2009 ambas rompieron su relación, yéndose la madre biológica de la vivienda que compartían y formulando demanda de divorcio.

Ante ello, la cónyuge de la madre biológica interpuso contra ésta demanda de reclamación de filiación por posesión de estado respecto de las gemelas, que fue estimado por el Juzgado de Primera Instancia, ordenándose la rectificación de las inscripciones de nacimiento de las menores, al efecto de hacer constar la filiación respecto de la actora y de que, consiguientemente, se modifiquen los apellidos de las menores. Recurrida dicha sentencia, la Audiencia Provincial desestimó el recurso, siendo interpuesto recurso de casación, que el Tribunal Supremo resuelve en la citada Sentencia.

La Sentencia critica, reproduciendo los correspondientes párrafos de la Sentencia de la Audiencia, que la reforma del Código Civil equiparando los matrimonios entre homosexuales y heterosexuales se haya hecho sin atender a otros aspectos en íntima relación con el matrimonio, como es el régimen legal de la filiación, en que las acciones de impugnación y reclamación estaban pensadas exclusivamente para parejas heterosexuales, sin mencionar las homosexuales, en que una de las personas no interviene en la fecundación, siendo la actual redacción del artículo 7 de la Ley 14/2006 la que permite a la cónyuge de la madre manifestar su consentimiento a la determinación a su favor de la filiación del nacido, pero para ello se exige que la manifestación se haga antes de que nazca el hijo, no en el momento de la inseminación, cuando además la filiación no puede quedar subordinada a un requisito formal como el consentimiento ante el encargado del Registro Civil, siendo así suficiente con el prestado ante la clínica una vez quede acreditado adecuadamente el voluntario 
consentimiento para la técnica de reproducción asistida y la voluntad concorde de las partes de concebir un hijo.

En el marco de la filiación por aplicación de estas técnicas se relativiza el principio de la verdad biológica, sustituyéndose por la voluntad de quien desea ser progenitor, posibilitándose así la coexistencia de dos filiaciones a favor de personas del mismo sexo: una filiación materna biológica y una filiación no basada en la realidad biológica, sino en una pura ficción legal, ambas con los mismos efectos jurídicos que la filiación por naturaleza, una vez se hayan cumplimentado los requisitos expuestos, lo que implica que en orden al ejercicio de una acción de reclamación de filiación no sea necesaria la impugnación de la ya determinada, pues no es contradictoria con la que se establece por ley. Por otra parte, esa prestación del consentimiento para la práctica de las técnicas de reproducción asistida, en cuanto que voluntad libre y manifestada por ambas del deseo de ser progenitoras, viene a superar a la posesión de estado y constituye causa para otorgar la filiación jurídica, aunque no exista vínculo biológico, creándose así un título de atribución de la paternidad al amparo del artículo 131 del Código Civil.

\section{A MODO DE CONCLUSIONES}

De este modo, podemos ver, que en un fuerte proceso de evolución en la equiparación entre parejas heterosexuales y homosexuales, por vía tanto legislativa como jurisprudencial, es posible afirmar hoy una amplia posibilidad de acceso de la mujer que sea pareja matrimonial o de hecho de otra mujer a la filiación de los hijos de ésta, ya sea por medio del recurso al expediente de adopción, conjunta o sucesiva, como mediante la atribución de la filiación por naturaleza en el caso de lesbianas casadas que recurren a las técnicas de reproducción asistida, posibilitándose en tal caso incluso el ejercicio de las acciones de filiación, en que lo relevante sería la prestación libre y conjunta del consentimiento para la práctica de las técnicas como manifestación de una posesión de estado.

Quedan no obstante muchos ámbitos en que esa equiparación dista mucho de ser real, como es el de las parejas de hecho o la concepción producida por vías distintas de las técnicas médicas de reproducción asistida, casos en que sólo cabe el recurso a la adopción. 


\section{Bibliografía}

- $\quad$ Arechederra, L. (2006). Decirle a dos personas mamá es muy difícil. Recuperado el 5 de febrero de 2014, de http://www.unav.es/cdb/undecirmama.html.

- Benavente Moreda, P. (2011), La filiación de los hijos de parejas, casadas o unidas de hecho, del mismo sexo. La situación legal y jurisprudencial actual. ADC, 64 (1), 75-124.

- Durán Ayago, A. (2004). Comentario al auto del Juzgado de Primera Instancia núm. 3 (familia) de Pamplona, de 22 de enero de 2004. Diario La Ley, (5.994), 1-5.

- Garaizabal, C. et al. (2012, 14 de diciembre). La maternidad lesbiana y sus derechos. Los nuevos modelos familiares avanzan a golpe de sentencias. El País, 14-12-2012. Recuperado el 5 de febrero de 2014 , de http://sociedad.elpais.com/sociedad/2012/12/14/actualidad/1355510303_479412.ht $\mathrm{ml}$.

- Gavidia Sánchez, J. V. (2001). Las uniones libres en la Ley Foral Navarra de Parejas Estables. En Actualidad Civil, (2), 605-643.

- Hernández Ibáñez, C. (2007). Repercusiones de la nueva Ley sobre Técnicas de Reproducción Humana Asistida, en materia de filiación". Revista Jurídica de la Universidad Interamericana de Puerto Rico, 41 (1 y 2), 885-897.

- Jiménez Muñoz, F. J. (2007). Observaciones sobre la nueva Ley española de técnicas de reproducción humana asistida". En Revista Jurídica de la Universidad Interamericana de Puerto Rico, 41, (1 y 2), 829-845.

(2008). Unas reflexiones sobre la nueva Ley de técnicas de reproducción humana asistida de 2006. Revista General de Legislación y Jurisprudencia, III Época, (4), 639-698.

(2012). La reproducción asistida y su régimen jurídico.

Madrid: Reus.

- Vallés Amores, M. L. (2005). La adopción. Exigencias subjetivas y su problemática actual. Madrid: Dykinson.

- Zurita Martín, I. (2006). Reflexiones en torno a la determinación de la filiación derivada de la utilización de las técnicas de reproducción asistida por una pareja de mujeres. Diario La Ley, (6.427), 1-5. 